\title{
Matériáta
}

ISSN 1517-7076

Revista Matéria, v. 11, n. 2, pp. 162 - 173, 2006

http://www.materia.coppe.ufrj.br/sarra/artigos/artigo10590

\section{Influencia del Fillers Calcáreo en la Solidificación/Estabilización de Metales Pesados}

\author{
TREZZA, Mónica A.
}

Facultad de Ingeniería, Universidad Nacional del Centro de la Provincia de Buenos Aires

Av. del Valle 5737 - Olavarría (B7400JWI)- Argentina Tel/fax: 54- 2284- 451055.

e-mail: mtrezza@,fio.unicen.edu.ar

\section{RESUMEN}

El cemento portland juega un rol cada vez más importante en el manejo de residuos industriales peligrosos. La técnica de solidificación/estabilización $(\mathrm{S} / \mathrm{S})$ basada en el cemento ha demostrado ser efectiva para el aislamiento de residuos peligrosos, especialmente metales pesados.

En este trabajo se discutirá el efecto que la adición de filler calcáreo al cemento produce en la S/S de metales como cromo, cadmio y plomo. Se realizaron estudios de difracción de rayos X, espectroscopia infrarroja, calorimetría y lixiviación a fin de evaluar el nivel de inmovilización en morteros conteniendo estos elementos. Se encontró un alto grado de inmovilización de los iones $\mathrm{Cd}, \mathrm{Cr}$ y $\mathrm{Pb}$ aunque su presencia modifica la velocidad y los compuestos obtenidos durante la hidratación del cemento.

Los resultados muestran que el cemento con fillers calcáreo es más eficiente que el cemento normal para la satisfactoria inmovilización de metales pesados.

Palavras chaves: Fillers calcáreo, cromo, cadmio, plomo, cemento.

\section{Influence of Limestone Fillers in Heavy Metals Solidification/Stabilization}

\section{ABSTRACT}

Portland cement plays an ever more important role in the management of hazardous industrials wastes. Cement - based solidification/stabilization (S/S) technique has been effectively demonstrated as a treatment to isolate hazardous waste especially heavy metals.

In the presents work will be discuss the effect that limestone filler addition to portland cement produced in the $\mathrm{S} / \mathrm{S}$ of metals as chromium, cadmium and lead. X-ray diffraction, infrared spectroscopy, calorimetric and leaching test were carried out to evaluate the level of immobilization in mortars made containing these elements. It was found that the degree of $\mathrm{Cd}, \mathrm{Cr}$ and $\mathrm{Pb}$ ion immobilization was very high but modifies the rate and the components obtains during the cement hydration.

The results thus presented prove that limestone blended cements material can be successfully used as a heavy metals immobilizing agent rather than the normal portland cement.

Keywords: Limestone filler, chromium, cadmium, lead, cement.

\section{$1 \quad$ INTRODUCCIÓN}

Los nuevos cementos conteniendo adiciones minerales activas (cenizas volantes, escorias y/o puzolanas) han demostrado una gran eficiencia en cuanto a su contribución a la durabilidad. El refinamiento de poros, la dilución del $\mathrm{C}_{3} \mathrm{~A}^{1}$ y la remoción del $\mathrm{CH}$ que producen son las principales ventajas que aportan, ventajas que a su vez favorecen su utilización en la solidificación/estabilización (S/S) de sustancias peligrosas, entre ellas metales como cromo, plomo y cadmio.

\footnotetext{
${ }^{1}$ Se utiliza la nomenclatura usual en la Química del cemento: C: $\mathrm{CaO}$, S: $\mathrm{SiO}_{2}, \mathrm{~A}: \mathrm{Al}_{2} \mathrm{O}_{3}, \mathrm{~F}: \mathrm{Fe}_{2} \mathrm{O}_{3}, \mathrm{H}: \mathrm{H}_{2} \mathrm{O}$ en consecuencia $\mathrm{C}_{3} \mathrm{~A}: 3 \mathrm{CaO} \cdot \mathrm{Al}_{2} \mathrm{O}_{3}, \mathrm{CH}: \mathrm{CaO} \cdot \mathrm{H}_{2} \mathrm{O}: \mathrm{Ca}(\mathrm{OH})_{2}$, y así siguiendo.
} 
La complejidad de la química de hidratación del cemento portland, sumada a la química de cada uno de los metales pesados a tratar, es un impedimento para comprender acabadamente las razones de su estabilización en matrices cementicias.

De todas formas, varios son los estudios de inmovilización de $\mathrm{Cr}(\mathrm{VI})$ en la matriz de cemento portland [1-4]. J Pera [ㅌ] informa que la inmovilización ocurre por alguno de los siguientes mecanismos o combinación de ellos: adición (ej. Cr-C-S-H), sustitución (ej. Cr-ettringita) ó precipitación de nuevos compuestos.

Otros autores estudiaron el comportamiento del sistema en presencia de $\mathrm{Cd}$ reportando la formación hidroxocadmiatos, resultantes de la reacción de $\left[\mathrm{Cd}(\mathrm{OH})_{4}\right]^{2-}$ con los iones $\mathrm{Ca}[\underline{6}]$ o la formación $\mathrm{de} \mathrm{Cd}(\mathrm{OH})_{2}$ y $\mathrm{Ca} / \mathrm{Cd}(\mathrm{OH})_{2}[\underline{7}-\underline{10}]$.

Por su parte, ha sido estudiada la estabilización del $\mathrm{Pb}$ en cemento común y de escoria granulada de alto horno [10]. Murat et al. reportan que sólo una pequeña cantidad de $\mathrm{Pb}$ es atrapada en el cemento normal, siendo más eficiente el cemento de alta alúmina [11]. Sin embargo no se han reportado fases cristalinas que contengan este elemento []].

Con la intensión de ampliar el espectro de matrices solidificantes y aprovechar la propiedades de las diferentes adiciones, incorporamos en este estudio el cemento con fillers calcáreo (caliza finamente dividida). Esta adición considerada inactiva, aunque hay claras evidencias de su reactividad, genera cambios diferentes a los determinados por las demás adiciones.

El efecto que produce la incorporación de caliza finamente molida es principalmente físico y su presencia incrementa el grado de hidratación de los principales componentes del clinker. A medida que aumenta la cantidad y finura de la caliza acelera la hidratación del $\mathrm{C}_{3} \mathrm{~S}$ y el C-S-H la incorpora en parte en su estructura. El filler provee nuevos centros de nucleación del $\mathrm{CH}$ [12] y genera la formación de carboaluminatos de calcio como consecuencia de la reacción del $\mathrm{CaCO}_{3}$ de la caliza con el $\mathrm{C}_{3} \mathrm{~A}$ del clinker portland [13]. Todo esto determina una aceleración de la hidratación temprana y una estructura más durable y en consecuencia potencialmente apta para la $\mathrm{S} / \mathrm{S}$ de sustancias peligrosas.

Se discute aquí la capacidad de este cemento para la estabilización de los iones metálicos $\mathrm{Cr}, \mathrm{Cd}$ y $\mathrm{Pb}$ y los posibles mecanismos de incorporación.

Los residuos metálicos se simularan con soluciones $0.1 \mathrm{M}$ de cromo hexavalente y $\mathrm{Cd} \mathrm{y} \mathrm{Pb}$ divalente. La evolución de la hidratación fue seguida por calorimetría diferencial, agua combinada y difracción de rayos X (DRX). Permitiendo esta última técnica la identificación de la $\mathrm{Cr}$ - ettringita como nueva fase de hidratación al incorporar $\mathrm{Cr}(\mathrm{VI})$ a cemento. Se realizaron además, ensayos de lixiviación a fin de evaluar el nivel de inmovilización de estos metales en morteros. En todo los casos se confrontarán los resultados con los obtenidos con el cemento sin fillers calcáreo.

Presentamos resultados que muestran que el cemento portland con fillers calcáreo es un lugar potencialmente adecuado para la estabilización de algunos metales pesados, aún con mayor eficiencia que el cemento portland normal.

\section{PROGRAMA EXPERIMENTAL}

\section{$2.1 \quad$ Materiales}

El cemento Portland de referencia $(\mathrm{C} 0)$ y el cemento con fillers calcáreo (C20) usados en este estudio, se obtuvieron por molienda conjunta a partir del mismo clinker portland. Dicho clinker contiene $58.2 \%$ de $\mathrm{C}_{3} \mathrm{~S}, 17,6 \%$ de $\mathrm{C}_{2} \mathrm{~S}$ y $14,7 \%$ de $\left(\mathrm{C}_{3} \mathrm{~A}+\mathrm{C}_{4} \mathrm{AF}\right)$, el contenido de caliza es de $19 \%$ en peso para $\mathrm{C} 20$.

Cantidades adecuadas de $\mathrm{C} 0$ y $\mathrm{C} 20$ fueron hidratadas con $\mathrm{w} / \mathrm{c}=0,4$ utilizando solución $0,1 \mathrm{M}$ de $\mathrm{Cd}(\mathrm{II}), \mathrm{Pb}(\mathrm{II})$ como nitratos y $\mathrm{Cr}(\mathrm{VI})$ como dicromato. Las muestras se mantuvieron selladas durante el tiempo de hidratación establecido. Dicha hidratación fue detenida por molienda con acetona. Las edades de ensayo fueron 1 hora, 1, 7, 28, 90, 180 días, 1 y 2 años.

\subsection{Estudios por DRX y FT-IR de las Muestras Hidratadas.}

Los difractogramas se registraron en el intervalo de 10 a $60^{\circ}$ de $2 \theta$, para ello se utilizó un equipo Philips PW 3710, con ánodo de cobre. Las medidas se realizaron usando un espectrofotómetro a transformada de Fourier (FT-IR), Nicolet modelo Magna 500 con ópticas de CsI. Se utilizó la técnica de pastillas de $\mathrm{KBr}$ y los espectros se registraron entre 4000 y $400 \mathrm{~cm}^{-1}$.

\section{$2.3 \quad$ Lixiviación}

La lixiviación de los diferentes iones fue evaluada sobre muestras curadas (14 días) usando el ensayo TCLP (toxicity characteristics leaching procedures) sugerido por la EPA para estos casos. La 
determinación cuantitativa de los iones metálicos fue realizada colorimétricamente usando un equipo Lasa 20 con los siguientes límites de detección: 0.03-1.0 mg/l Cr, $0.1-0.2 \mathrm{mg} / 1 \mathrm{~Pb}$ y $0.02-0.3 \mathrm{mg} / 1 \mathrm{Cd}$. La solución de lixiviación fue agua y solución ácida $(\mathrm{pH}=3)$ y el tiempo de extracción 18 horas.

\subsection{Agua Combinada}

Probetas de pasta $(\mathrm{w} / \mathrm{c}=0.4)$ fueron usadas para la determinación del contenido de agua combinada (Wn). Wn fue obtenida de acuerdo al procedimiento propuesto por Powers [14]. Este valor se usó como medida para estimar el grado de hidratación $(\alpha)$ suponiendo que el filler es una adición químicamente no activa y el agua necesaria para la total hidratación de $1 \mathrm{~g}$ de cemento es $0.23 \mathrm{~g}$.

\subsection{Calorimetría Diferencial}

Las determinaciones de calorimetría diferencial se realizaron con un equipo de fabricación propia de la Facultad de Ingeniería - UNCPBA que consta de las siguientes partes: dos recipientes Dewar aislados térmicamente, un termopar diferencial cobre-constantan del tipo T, todo aislado adecuadamente y con control de temperatura. Finalmente todo el equipo se conecta a un sistema electrónico de adquisición de datos.

\section{RESULTADOS Y DISCUSION}

\subsection{Estudio por DRX y FT-IR de las Muestras Hidratadas.}

\subsubsection{Cromo}

Bensted y Prakash Varma [15] informan que el $\mathrm{CrO}_{4}{ }^{2-}$ puede sustituir al $\mathrm{SO}_{4}{ }^{2-}$ formando la cromoettringita: $\left(3 \mathrm{CaO} . \mathrm{Al}_{2} \mathrm{O}_{3} \cdot 3 \mathrm{CaCrO}_{4} \cdot 32 \mathrm{H}_{2} \mathrm{O}\right)$ ya que durante la hidratación del cemento muchos iones $\mathrm{CrO}_{4}{ }^{2-}$ tienden a congregarse en la fase ettringita $\left(3 \mathrm{CaO} \cdot \mathrm{Al}_{2} \mathrm{O}_{3} \cdot 3 \mathrm{CaSO}_{4} \cdot 32 \mathrm{H}_{2} \mathrm{O}\right)$.

Según dichos autores la Cr-ettringita tiene estructura cristalina similar a la ettringita y en consecuencia sus patrones de DRX presentan grandes similitudes. En particular la Cr-ettringita se caracteriza por el corrimiento de los picos de la ettringita y la aparición de un pico característico en $\mathrm{d}=4,71 \AA$ ( $\mathrm{d}=5.70$, 4.71 y $3.52 \AA)$.

En C20 hidratada con solución de Cr(VI) a la edad de 90 días (Figura 1), se observa un corrimiento de los principales picos de la ettringita y la aparición de un pico en $\mathrm{d}=4,75 \AA(18,66$ de $2 \theta)$ que indican la incorporación de cromo a la estructura cristalina por formación de Cr-ettringita. En las muestras de C0 hidratadas con $\mathrm{Cr}(\mathrm{VI})$ no se detectaron los corrimientos mencionados para C20. Esto parece indicar que, a esta edad de hidratación, el cemento con fillers incorpora parcialmente el cromo en su estructura por formación de esta fase estable.

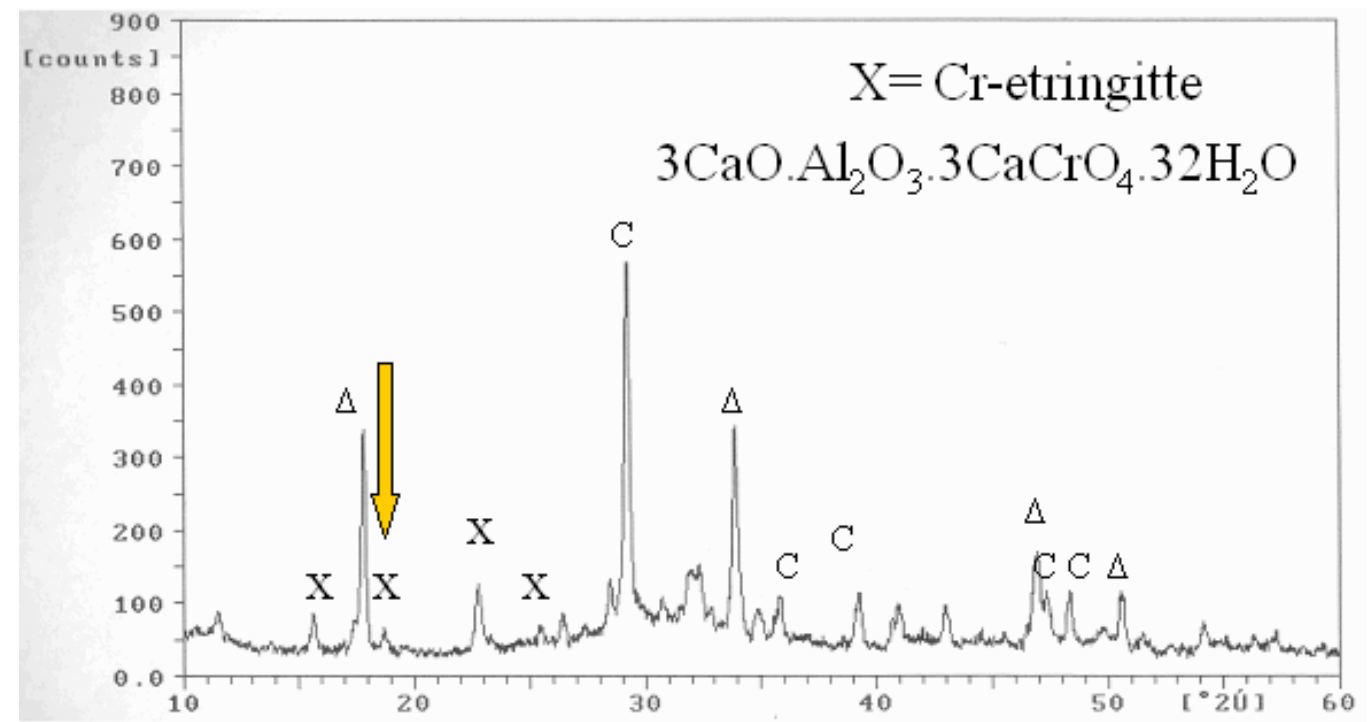

Figura 1: Difractograma C20 hidratado en presencia de $\mathrm{Cr}(\mathrm{VI})$ a la edad de 180 días. 
La presencia de caliza presupone la formación de carboaluminatos durante la hidratación del cemento. Dentro de ellos el monocarboaluminato es la fase más estable, y ha podido ser identificada en las muestras hidratadas con agua y con solución de cromo. Sin embargo la fase formada en presencia de $\mathrm{Cr}(\mathrm{VI})$ no ha sido identificada totalmente hasta los 180 días de hidratación.

En trabajos previos [16] se realizaron análisis cuantitativos, a partir de los espectros de DRX utilizando uno de los picos principales de la caliza $2 \theta=29^{\circ}$, a fin de determinar el porcentaje de caliza incorporado a las fases hidratadas. Los mismos nos permiten corroborar que la caliza incorporada al sistema en presencia de cromo es menor en toda edad inferior a 6 meses. A partir de esa edad el consumo aumenta considerablemente con respecto a la referencia por formación de monocarboaluminato de calcio ya mencionado.

\subsubsection{Cadmio}

Cuando C0 se hidrató en la presencia de Cd(II) no se detectaron por DRX compuestos de hidratación diferentes a los normalmente presentes en la hidratación del cemento normal, señalando que la presencia de $\mathrm{Cd}(\mathrm{II})$ en el sistema no determina modificaciones significativas.

Para el caso de C20 hidratada con solución de Cd(II) en cambio, se observa un corrimiento de los picos de $\mathrm{Ca}(\mathrm{OH})_{2}$ hacia mayores valores de $\mathrm{d}$, como se puede apreciar en la Tabla 1 .

Tabla 1: Seguimiento por DRX del hidróxido de calcio en muestras hidratadas en presencia de Cd(II)

\begin{tabular}{|c|c|c|c|c|}
\hline \multirow{2}{*}{$\begin{array}{c}\text { Datos bibliografía [17] } \\
\text { (principales picos) }\end{array}$} & \multicolumn{2}{|c|}{ C0 } & \multicolumn{2}{c|}{ C20 } \\
\cline { 2 - 5 } & 28 días & 90 días & 28 días & 90 días \\
\hline 4.90 & 4.9038 & 4.8918 & 4.9514 & 4.9432 \\
\hline 2.628 & 2.6275 & 2.6268 & 2.6407 & 2.6392 \\
\hline 1.927 & 1.9279 & 1.9225 & 1.933 & 1.9328 \\
\hline 1.796 & 1.7958 & 1.7967 & 1.7732 & 1.7726 \\
\hline
\end{tabular}

En el caso de $\mathrm{C} 20$, es posible que el corrimiento de los picos de $\mathrm{CH}$ se deba al hecho de la formación de solución sólida entre el $\mathrm{Ca}$ y el $\mathrm{Cd}$ y/o formación de un hidróxido mixto $(\mathrm{Ca}, \mathrm{Cd})(\mathrm{OH})_{4}$ por interacción del $\mathrm{Ca}^{2+}$ con el anión $\left[\mathrm{Cd}(\mathrm{OH})_{4}\right]^{2-}$ debido al alto $\mathrm{pH}$ del medio $[\underline{18}]$.

Con el tiempo de hidratación el parámetro disminuye y se acerca al del $\mathrm{CH}$ puro, seguramente se deba a que se afianza la formación del $\mathrm{Cd}(\mathrm{OH})_{2}$ puro. Efectivamente a edades avanzadas de hidratación (24 meses) se detectó la presencia de $\mathrm{Cd}(\mathrm{OH})_{2}$ por aparición de nuevos picos $(\mathrm{d}=4.70,3.03,2.55 \AA)$.

En las muestras de $\mathrm{C} 0$ hidratadas con $\mathrm{Cd}(\mathrm{II})$ no se detectaron los corrimientos mencionados para C20 ni los picos característicos del hidróxido de cadmio. Esto parece indicar que el cemento con fillers, a diferencia que el cemento normal, estabiliza el cadmio en su estructura por formación de compuestos estables.

En cuanto a la formación de monocarboaluminato la presencia de $\mathrm{Cd}(\mathrm{II})$ en la hidratación de $\mathrm{C} 20$ no lo inhibe. El mismo se ha detectado (su pico principal) por DRX a edades mayores a 28 días.

\subsubsection{Plomo}

Los difractogramas correspondientes a las muestras hidratadas en presencia de solución de $\mathrm{Pb}(\mathrm{II})$ muestran claramente el atraso de la hidratación respecto a las restantes muestras. Sin embargo cabe destacar que existe una considerable diferencia entre $\mathrm{C} 0$ y C20. El efecto fillers en C20 determina esta diferencia de velocidad de hidratación que la presencia de plomo amortigua pero no anula.

No se han detectado, por esta técnica, fases en las cuales intervenga el plomo.

\subsubsection{Estudios por Espectroscopia Infrarroja}

Las mediciones de los espectros FT-IR confirmaron la información ya discutida respecto a la modificación de las velocidades de hidratación temprana por la presencia de los metales incorporados. A modo de ejemplo la Figura 2 muestra el espectro correspondiente a C20 hidratado en presencia de cadmio después de 1 hora y 28 días de hidratación. 


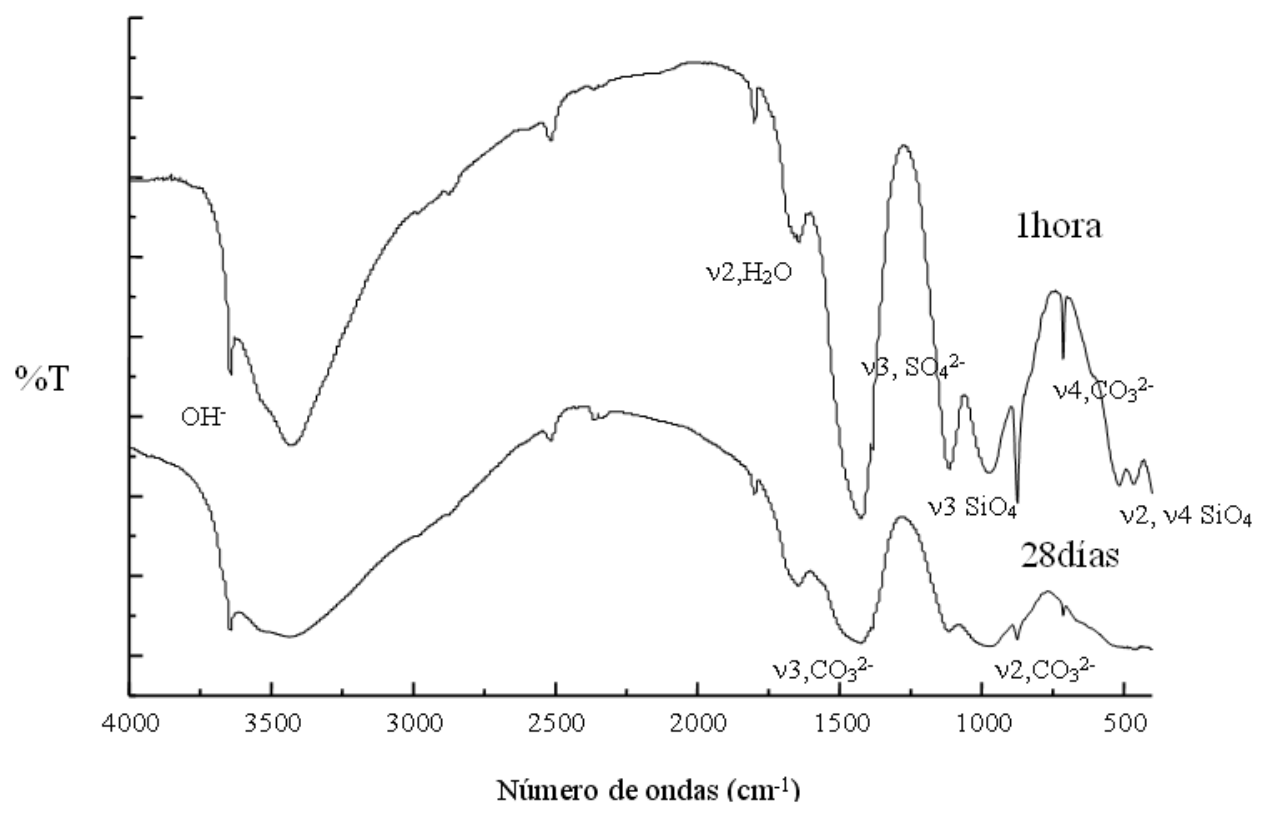

Figura 2: Espectro FT-IR C20 hidratado en presencia de $\mathrm{Cr}(\mathrm{VI})$ a la edad de 1 hora y 28 días .

El avance de la hidratación es seguido por esta técnica a través de la aguda banda en $3645 \mathrm{~cm}^{-1}$ cuya intensidad crece con el tiempo de hidratación. La misma es asignable a los $\mathrm{OH}^{-} \operatorname{del~} \mathrm{Ca}(\mathrm{OH})_{2}$ formado [1921]. En la zona 3100- $3400 \mathrm{~cm}^{-1}$ aparece una banda ancha debida a los estiramientos simétricos y antisimétricos $\left(\begin{array}{lll}v & \mathrm{y} & \mathrm{v} 3\end{array}\right)$ del vibrador $\mathrm{O}-\mathrm{H}$ de las moléculas de agua. Por su parte la banda a $1650 \mathrm{~cm}^{-1}$ aproximadamente es el modo deformación H-O-H ( $v 2)$ del agua molecular adsorbida.

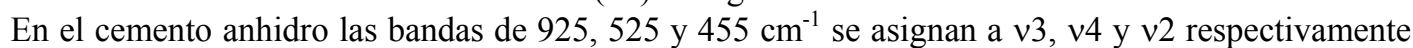
del grupo $\mathrm{SiO}_{4}{ }^{4-}$. La característica principal en las muestras hidratadas es el corrimiento del modo estiramiento $v 3$ por más de $50 \mathrm{~cm}^{-1}$ debido a la polimerización de las unidades $\mathrm{SiO}_{4}{ }_{4}^{4-}$. Esto sumado al cambio en las intensidades relativas de $\vee 2$ y $v 4$ constituye una clara evidencia del grado de polimerización alcanzado con la formación de la fase C-S-H como resultado de la hidratación del cemento [20].

Las banda de 1420,872 y $712 \mathrm{~cm}^{-1}$ se asignan a $v 3, v 2$ y $v 4 \mathrm{CO}_{3}^{-2}$ respectivamente. Las mismas se encuentran en el cemento anhidro y se asignan a la calcita agregada como consecuencia de la incorporación del filler. Dichas bandas persisten en la medida que la adición permanezca inerte en el sistema. En cuanto al monocarboaluminato de calcio su presencia se detecta mediante FT-IR por el desdoblamiento de $23 \mathrm{CO}_{3}{ }^{2-}$ en $1365-1426 \mathrm{~cm}^{-1}$ y la aparición de un hombro en $1066 \mathrm{~cm}^{-1}$ asignable a $v 1 \mathrm{CO}_{3}{ }^{2-}$ señalando que la simetría del grupo carbonato disminuye en el monocarboaluminato [22-23]. Si bien con el avance de la hidratación vemos que $v 3 \mathrm{CO}_{3}{ }^{2-}$ se deforma, a esta edad de hidratación y a través de esta técnica, no hay evidencias claras de la formación del compuesto.

El yeso adicionado se asigna sobre la base del grupo $\mathrm{SO}_{4}{ }^{2-}$ de simetría tetraédrica, en consecuencia sólo presentan modos activos en IR la deformación fuera del plano v4 en $600 \mathrm{~cm}^{-1}$ y el doblete 1120 $1145 \mathrm{~cm}^{-1}$ asignable a $v 3 \mathrm{SO}_{4}{ }^{2-}$. Este doblete se transforma en una única banda centrada en $1120 \mathrm{~cm}^{-1}$ como consecuencia de la formación de ettringita $\left(3 \mathrm{CaO} \cdot \mathrm{Al}_{2} \mathrm{O}_{3} \cdot 3 \mathrm{CaSO}_{4} \cdot 32 \mathrm{H}_{2} \mathrm{O}\right)$ durante la hidratación.

\subsection{Lixiviación}

\subsubsection{Cromo:}

Los resultados de los ensayos de lixiviación realizados en agua y a $\mathrm{pH}=3$ sobre el cemento $\mathrm{C} 0$ y $\mathrm{C} 20$ hidratados con $\mathrm{Cr}(\mathrm{VI})$ a diferentes edades, se muestran en la Tabla 2. 
Tabla 2: Concentración de $\mathrm{Cr}(\mathrm{VI})$ obtenida por lixiviación, a diferentes edades. Concentración inicial: 2080 ppm

\begin{tabular}{|c|c|c|c|c|}
\hline $\mathrm{CO}$ & \multicolumn{2}{|c|}{ Lixiviación en agua } & \multicolumn{2}{|c|}{ Lixiviación en ácido } \\
\hline Edad & ppm & $\%$ retenido & ppm & $\%$ retenido \\
\hline $1 \mathrm{mes}$ & 208 & 90.0 & 604 & 71.0 \\
\hline 3 meses & $50 ?$ & 97.6 & 420 & 79.8 \\
\hline 6 meses & 72 & 96.5 & 430 & 79.3 \\
\hline 12 meses & 80 & 96.1 & 440 & 78.8 \\
\hline 24 meses & 88 & 95.8 & 360 & 82.7 \\
\hline C20 & \multicolumn{2}{|c|}{ Lixiviación en agua } & \multicolumn{2}{|c|}{ Lixiviación en ácido } \\
\hline Edad & ppm & $\%$ retenido & ppm & $\%$ retenido \\
\hline $1 \mathrm{mes}$ & 128 & 93.9 & 650 & 68.8 \\
\hline 3 meses & 124 & 94.0 & 510 & 75.5 \\
\hline 6 meses & 132 & 93.7 & 630 & 69.7 \\
\hline 12 meses & 132 & 93.7 & 760 & 63.5 \\
\hline 24 meses & 120 & 94.2 & 620 & 70.2 \\
\hline
\end{tabular}

Para el C0 la cantidad de $\mathrm{Cr}(\mathrm{VI})$ total lixiviado disminuye rápidamente entre 1 y 3 meses de hidratación. A medida que aumentan los productos de hidratación, con mayor área superficial y por mayor densificación de la microestructura, más iones son atrapados y estabilizados químicamente [3]].

Para toda edad de hidratación el contenido de $\mathrm{Cr}(\mathrm{VI})$ retenido es mayor cuando el ensayo se realiza en agua que a $\mathrm{pH}=3$.

Cuando se trabaja con C20 el porcentaje de cromo retenido después de la lixiviación en agua se mantiene prácticamente constante para toda edad de hidratación, siendo los mismos inferiores que para C0. Sin embargo es bueno recordar que C20 tiene un porcentaje de reemplazo de $18-19 \%$; en consecuencia la cantidad de cemento disponible es menor, lo que muestra una mayor eficiencia de retención del $\mathrm{Cr}(\mathrm{VI})$ por el cemento en presencia de fillers calcáreo. Esto concuerda con lo observado anteriormente; la formación de productos de hidratación a edades más tempranas y de compuestos cristalinos que incorporan al cromo determina este comportamiento. La misma situación se repite para la lixiviación en medio ácido.

\subsubsection{Cadmio}

Si bien las probetas ensayadas poseían $4496 \mathrm{ppm}$ de $\mathrm{Cd}(\mathrm{mgCd} / \mathrm{Kg}$ cemento) las determinaciones realizadas no detectaron su presencia. Esto indica que la concentración de $\mathrm{Cd}(\mathrm{II})$ en el lixiviado es menor de $0.02 \mathrm{mg} / \mathrm{l}$ lo que significa, basándose en el ensayo realizado menos de $0.4 \mathrm{mgCd}(\mathrm{II}) / \mathrm{Kg}$ cemento, o sea una reducción de aproximadamente 10.000 veces. Estos resultados ponen de manifiesto la gran capacidad del cemento portland y del cemento con filler calcáreo para la estabilización del cadmio.

\subsubsection{Plomo}

Nuevamente se observa aquí que la capacidad del C0 para retener el metal aumenta conforme avanzan los procesos de hidratación. En tanto que para C20 el porcentaje retenido se mantiene prácticamente invariable para toda edad de hidratación. De todas formas ambos cementos, independientemente del medio de lixiviación, son sumamente aptos para la retención del ión $\mathrm{Pb}(\mathrm{II})$ tal como se deduce de los altos porcentajes de retención obtenidos. 
Tabla 3: Concentración de $\mathrm{Pb}(\mathrm{II})$ obtenida por lixiviación, a diferentes edades.

Concentración inicial: 8288 ppm

\begin{tabular}{|c|c|c|c|c|}
\hline $\mathrm{CO}$ & \multicolumn{2}{|c|}{ Lixiviación en agua } & \multicolumn{2}{|c|}{ Lixiviación en ácido } \\
\hline Edad & ppm & $\%$ retenido & ppm & $\%$ retenido \\
\hline $1 \mathrm{mes}$ & 28.6 & 99.7 & 11.4 & 99.8 \\
\hline 3 meses & 18.4 & 99.8 & 17.6 & 99.8 \\
\hline 6 meses & $8.8 ?$ & 99.9 & 14.0 & 99.8 \\
\hline 12 meses & 16.4 & 99.8 & 13.2 & 99.8 \\
\hline 24 meses & 12.2 & 99.8 & 3.4 & 99.9 \\
\hline C20 & \multicolumn{2}{|c|}{ Lixiviación en agua } & \multicolumn{2}{|c|}{ Lixiviación en ácido } \\
\hline Edad & ppm & $\%$ retenido & ppm & $\%$ retenido \\
\hline $1 \mathrm{mes}$ & 46.0 & 99.4 & 15.8 & 99.8 \\
\hline 3 meses & 24.0 & 99.7 & - & - \\
\hline 6 meses & - & - & 15.0 & 99.8 \\
\hline 12 meses & 18.2 & 99.8 & 15.6 & 99.8 \\
\hline 24 meses & 8.2 & 99.9 & 7.4 & 99.9 \\
\hline
\end{tabular}

\subsection{Agua Combinada}

La evolución de las propiedades mecánicas de los diferentes sistemas estudiado está asociado con la cinética de hidratación de los cementos. La Figura 3 muestra la evolución del grado de hidratación a través del contenido de agua combinada para los distintos cementos y condiciones de hidratación.

Los resultados obtenidos por este método no son muy precisos, pero son índices suficientes del progreso de las reacciones de hidratación y así se han utilizado satisfactoriamente [24]. La presencia de fillers calcáreo (C20) acelera la hidratación temprana con respecto al cemento portland de referencia (C0) como era de esperar, independientemente de la presencia de $\mathrm{Cd}, \mathrm{Cr}$ y $\mathrm{Pb}$.

El cromo es de los metales incorporados quien menos afecta la hidratación temprana del cemento, siendo su evolución aproximadamente igual a la esperada para el cemento sin la presencia del metal. Sin embargo su efecto es más pronunciado en el cemento con fillers donde su evolución es ligeramente inferior a partir de 7 días de hidratación. La formación de Cr-ettringita y el retraso en la formación del monocarboaluminato de calcio determinan este efecto, tal como se discutió a través de los DRX.

Con respecto a la presencia de plomo y cadmio esta siempre retrasa la hidratación temprana (especialmente el plomo), sin embargo este efecto se minimiza a edades mayores a 28 días. 

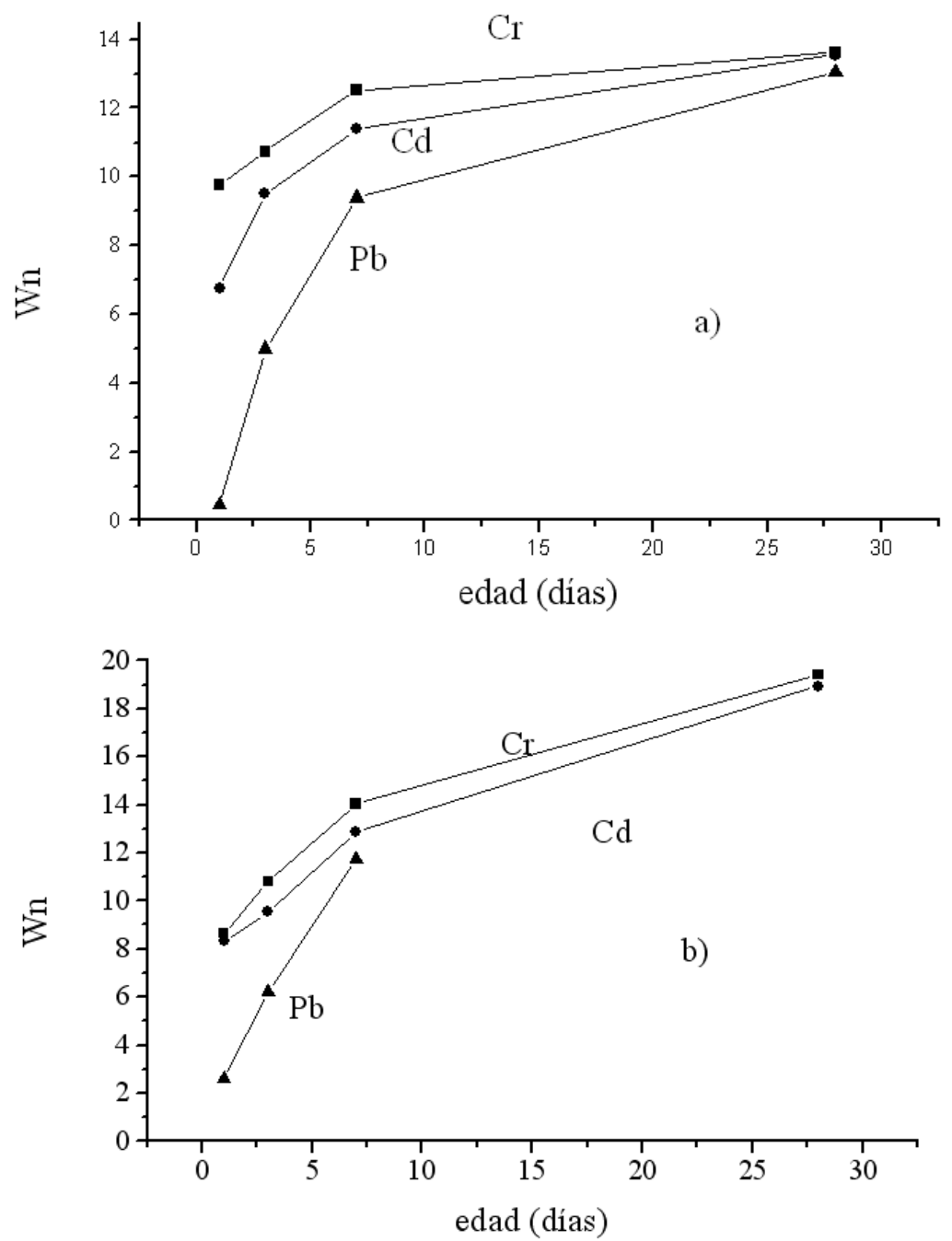

Figura 3: Agua combinada vs edad de hidratación de las muestra hidratadas con soluciones de $\mathrm{Cr}(\mathrm{VI}), \mathrm{Cd}(\mathrm{II})$ y $\mathrm{Pb}(\mathrm{II})$. a) $\mathrm{C} 0 \quad$ b) $\mathrm{C} 20$

\subsection{Calorimetría Diferencial}

Las curvas; temperatura vs tiempo; obtenidas para los diferentes sistemas en estudio difieren significativamente unas de otras (situación que analizaremos posteriormente), pero en primer lugar se discutirá la forma general de las curvas y las zonas que quedan determinadas.

La Figura 4 esquematiza una curva calorimétrica típica de un cemento normal en el cual se pueden distinguir las siguientes 4 zonas. La primera de ellas se define tan pronto como el cemento se pone en contacto con el agua de hidratación. El calor desarrollado corresponde al mojado y solubilización superficial de las partículas [25]. El agua se satura de hidróxido de calcio producido por la hidrólisis del $\mathrm{C}_{3} \mathrm{~S}$ y el aluminato tricálcico. Conjuntamente a la disolución de estos iones, lo hacen también los hidróxidos alcalinos del clinker. La cinética de este proceso es rápida y el control químico. 


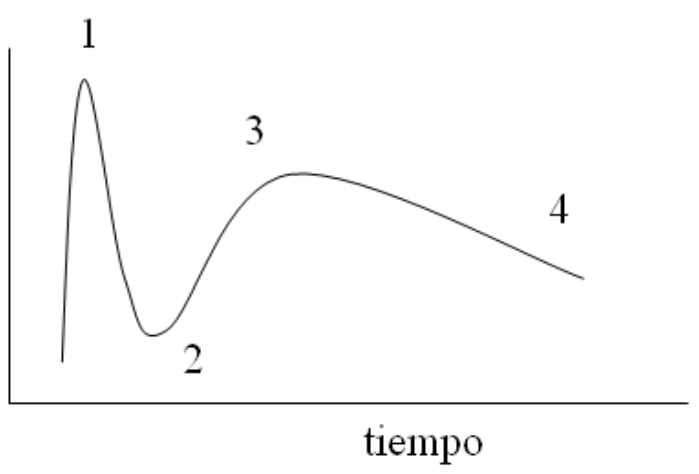

Figura 4: Esquema de una curva calorimétrica típica.

A esta etapa sigue un proceso de aparente inactividad, conocido como "período durmiente", esta etapa determina que el cemento permanezca en estado plástico por algunas horas. Desde el punto de vista químico, durante este período los iones se concentran en la disolución -formación de núcleos de tamaño crítico- antes de iniciarse la precipitación de los productos de hidratación. El final del período durmiente determina el inicio del fraguado.

Posteriormente se observa un período de aceleración que determina en su punto máximo el final del proceso de formación de núcleos de los hidratos y el inicio de la ganancia de resistencia de la pasta debido al crecimiento y entrecruzamiento de éstos últimos. Por ello este máximo se suele correlacionar con el final del fraguado [ㄷ]. Este proceso controlado químicamente se origina por la formación de productos de hidratación a partir de los silicatos.

Aparentemente [27], el CH cristaliza desde la solución, mientras que el C-S-H se desarrolla sobre la superficie de los granos de $\mathrm{C}_{3} \mathrm{~S}$, cubriéndolos. Así al continuar la hidratación crece la capa formada e interfiere, actuando como barrera, entre el material sin hidratar y el agua. Por ello los iones deben difundir para alcanzar el material anhidro. Esto determina un período de desaceleración controlado química y difusionalmente. El movimiento de los iones a través de las capas de C-S-H determina la velocidad de reacción de la cuarta etapa, la cual viene controlada por difusión. Estos procesos son más lentos a medida que crece el ancho de la barrera, en consecuencia se completan a muy largo plazo. Esta etapa determina la velocidad de ganancia de resistencia.

La contribución al calor de hidratación del $\mathrm{C}_{3} \mathrm{~A}$ no pudo ser distinguida en este estudio dado el bajo contenido del mismo en el cemento usado.

\subsubsection{Cromo}

La presencia de $\mathrm{Cr}$ en el agua de mezclado, no parece influir sobre la velocidad de hidratación temprana (primeras 48 horas) del cemento de referencia. Algo similar ocurre para C20. Tal como se observó a través de $\mathrm{Wn}$, esta técnica confirma que la incorporación de cromo al sistema, en las condiciones de este estudio, no afectan el principio y el fin del fraguado.

\subsubsection{Cadmio}

Al hidratar $\mathrm{C} 0$ y $\mathrm{C} 20$ en presencia de $\mathrm{Cd}(\mathrm{II})$ se observa que afecta sensiblemente dicha velocidad. En el caso de $\mathrm{C} 0$, la presencia de cadmio acorta el periodo durmiente y en consecuencia adelanta el principio y final del fraguado. Si el cemento usado es C20 este efecto se reduce a atrasar ligeramente el inicio del fraguado sin afectar el final del mismo. En este último caso se observa claramente como la etapa de hidratación inicial adquiere valores superiores a la referencia por la mayor actividad superficial causada por el filler (Figura 5-a). 

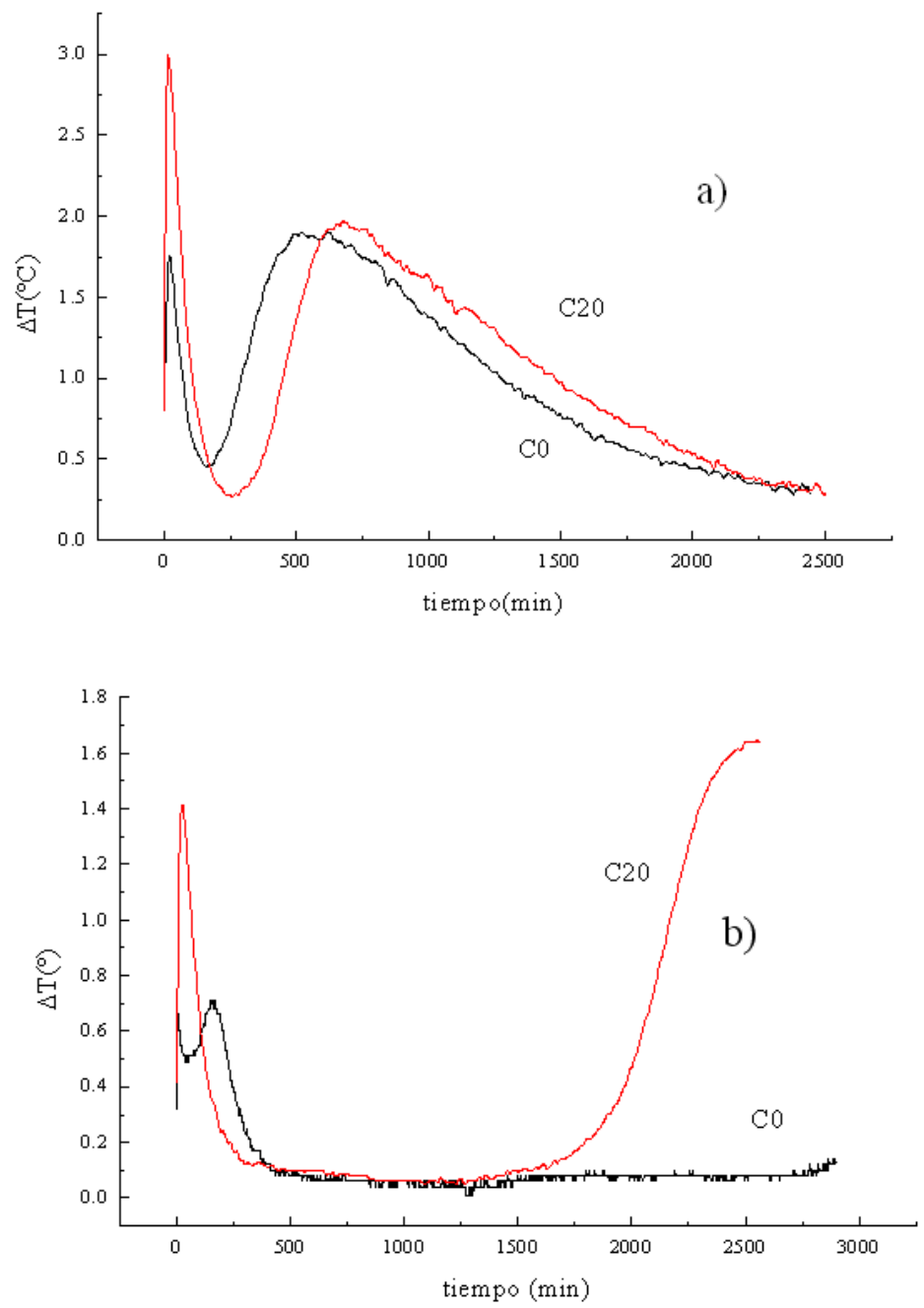

Figura 5: Curvas calorimétricas de $\mathrm{C} 0$ y $\mathrm{C} 20$. a) Hidratadas en presencia de $\mathrm{Cd}(\mathrm{II})$ b) Hidratadas en presencia de $\mathrm{Pb}(\mathrm{II})$

\subsubsection{Plomo}

Al hidratar las muestras $\mathrm{C} 0$ y $\mathrm{C} 20$ con solución de $\mathrm{Pb}(\mathrm{II})$ se observaron las siguientes diferencias. C0 mostró dos picos iniciales de hidratación, diferidos en el tiempo, señalando un retardo en las etapas de mojado y disolución. Estas etapas al estar ligadas a la superficie expuesta a la hidratación no se diferencian en C20, cuya superficie específica es mucho mayor por la presencia del filler (Figura 5-b).

Es de notar la gran diferencia encontrada en el tiempo de fraguado por la sola presencia del plomo. Sin embargo este retraso es mucho mas significativo en el caso de C0 que en C20. En C20 el inicio del fraguado se da a partir de las 25 horas de hidratación (1500 min.) y finaliza aproximadamente a las 42 horas (2500 min.).

Para $\mathrm{C} 0$, en cambio, el periodo durmiente se extiende por varios días de forma que el inicio del fraguado se observa recién a las 70 horas y el final se retarda aún más. El pico obtenido es chato y de baja altura (máximo $0,7^{\circ} \mathrm{C}$ ) pero muy extendido en el tiempo.

Esto nos indicaría que en C20 el plomo es extraído del sistema y en consecuencia su efecto sobre el tiempo de fraguado es menor. Evidentemente hay aquí otra prueba de la estabilización del contaminante en mayor proporción que en la referencia como consecuencia de la presencia del filler. 


\section{CONCLUSIONES}

La utilización de cemento portland con filler calcáreo en la $\mathrm{S} / \mathrm{S}$ de metales pesados condujo a los siguientes resultados:

- La aceleración de los procesos iniciales de hidratación como consecuencia de la presencia de fillers y la generación de un trama más cerrada a edades tempranas, explica el hecho que en el cemento con fillers sea una matriz muy adecuada para la $\mathrm{S} / \mathrm{S}$ de algunos metales pesados.

- En todos los casos el porcentaje de metal retenido en la estructura cementicia alcanzó altos porcentajes. Esto indica que, en las condiciones y tiempo de ensayo estudiados, el cemento muestra una interesante capacidad de retención, reduciendo los riesgos por lixiviación de cromo, cadmio y plomo y su consecuente efecto nocivo para la salud y/o el medio ambiente.

- El cromo y el cadmio se estabilizan en la matriz cementicia por formación de Cr-ettringita e hidróxido de cadmio respectivamente, fases estables e identificadas por DRX. Independientemente de las fases encontradas, los ensayos de lixiviación señalan una clara evidencia de la solidificación del cadmio y el cromo en la matriz de cemento.

- De plomo no se han identificado nuevas fases que expliquen su estabilización, pero la solidificación del mismo es evidente tal lo mostrado en los ensayos de lixiviación.

\section{BIBLIOGRAFIA}

[1] FRÍAS, M., SÁNCHEZ DE ROJAS, M.I., GARCÍA, N., LUXÁN, M.P., "Contribution of Toxic Elements: Hexavalent Chromium in Materials Used in the Manufacture of Cement", Cement and Concrete Research, v. 24, pp. 533-541, 1994.

[2] MACÍAS, A., KINDNESS, A., GLASSER, F.P., "Impact of Carbon Dioxide on the Immobilization Potential of Cemented Wastes: Chromium", Cement and Concrete Research, v. 27, pp. 215-225, 1997.

[3] PARK, C.-K., "Hydration and solidification of hazardous wastes containing heavy metals using modified cementitious materials", Cement and Concrete Research, v. 30, pp. 429- 435, 2000.

[4] WANG, S., VIPULANANDAN, C., "Solidification/stabilization of Cr(VI) with Cement Leachability and XRD Analices", Cement and Concrete Research, v. 30, pp. 385-389, 2000.

[5] PERA, J., THEVENIN, G., CHABANNET, M., "Design of a Novel System Allowing the Selection of an Adequate Binder for Solidification/Tabilization of Wastes", Cement and Concrete Research, v. 27, pp. 1536-1542, 1997.

[6] MOLLAH, M.Y.A., TSAI, Y.-N., COCKE, D.L., "An FTIR Investigation of Cement Based solidification/satbilization Systems Doped With Cadmium", Environmental Science and Health, A27, v. 5, pp. 1213-1227, 1992.

[7] DIEZ, J.M., MADRID, J., MACÍAS, A., "Characterization of Cement-stabilized Cd Wastes", Cement and Concrete Research, v. 27, pp. 479-485, 1997.

[8] TUMIDAJSKI, P.J., THOMSON, M.L., "Influence of Cadmium on the Hydration of $\mathrm{C}_{3} \mathrm{~A}$ ", Cement and Concrete Research, v. 24, pp. 1359-1372, 1994.

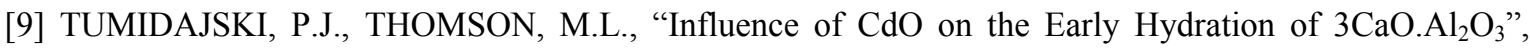
Cement and Concrete Research, v. 25, pp. 1690-1690, 1995.

[10] MADRID, J., DIEZ, J.M., GOÑI, S., MACÍAS, A., "Durability of Cement Matrices Used for Stabilization of Hazardous Wastes". In: International Conference Durability of Concrete, São Paulo, pp. 1527-1551, 1997.

[11] MURAT, M., SORRENTINO, F., "Effect of Large Additions of Cd, $\mathrm{Pb}, \mathrm{Cr}, \mathrm{Zn}$ to Cement Raw Meal on the Composition and the Properties of Clinker and the Cement", Cement and Concrete Research, v. 26, pp. 377-385, 1996. 
[12] SOROKA, I., STERN, W., "Calcareous Fillers and the Compressive Strength of Portlant Cement", Cement and Concrete Research, v. 6, n. 3, pp. 367-376, 1976.

[13] RAMACHANDRAN, V., ZHANG, C., "Hydration Kinetics and Microestructural Development in the $3 \mathrm{CaO} . \mathrm{Al}_{2} \mathrm{O}_{3}-\mathrm{CaSO}_{4} \cdot 2 \mathrm{H}_{2} \mathrm{O}-\mathrm{CaCO}_{3}-\mathrm{H}_{2} \mathrm{O}$ System", Materiales de Construccion, v. 19, n. 114, pp. 437-444, 1986.

[14] POWERS, T.C., "The non Evaporable Water Content of Hardened Portland Cement Pastes", ASTM Bull, n. 158, pp. 68- 75, 1949.

[15] BENSTED, J., PRAKASH VARMA, S., "Studies of Ettringite and its Derivatives: Part II. Chromate Substitution", Silicates Industriels, v. 37, n. 12, pp. 315-318, 1972.

[16] TREZZA, M.A., FERRAIUELO, M.F., "Hydration Study of Limestone Blended Cement in the Presence of Hazardous Wastes Containing Cr(VI)", Cement and Concrete Research, v. 33, pp. 1039-1045, 2003.

[17] TAYLOR, H.F.W., La Química del Cemento, v. II, Apéndice 1: Tabla de datos cristalográficos. Ed. Urmo, España, 1971.

[18] MOLLAH, M.Y., TSAI, Y-N, COCKE, D.L., "An FTIR Investigation of Cement Based solidification/stabilization Systems Doped with Cadmium”, Environmental Science and Health, A27, n. 5, pp. 1213-1227, 1992.

[19] ORIOL, M., PERA, J., "Pozzolanic Activity of Metakaolin Under Microwave Treatment", Cement and Concrete Research, v. 25, pp. 265-270, 1995.

[20] MOLLAH, M.Y., PALTA, P., HESS, T., et al., "Chemical ang Physical Effects of Lignosulfonate Superplasticizer on the Hydration of Portland Cement and solidification/stabilization Consequences”, Cement and Concrete Research, v. 25, pp. 971- 682, 1995.

[21] DUTTA, D., BORDOLOR, D., BOTHAKUR, P., "Hydration of Portland Cement Clinker in the Presence of Carbonaceous Materials”, Cement and Concrete Research, v. 25, n. 5, pp. 1095-1102, 1995.

[22] BENSTED, J., PRAKASH VARMA, S., "Some Applications of Infrared and Raman Spectroscopy in Cement Chemistry, Part 3”, Cement Technology, v. 5, n. 5, pp. 440-442, 1974.

[23] BENSTED, J., PRAKASH VARMA, S., "Infrares and Raman Spectroscopy in Cement Chemistry.Miscellaneous Applications”, World Cement, v. 8, n. 1, pp. 16, 18-20, 1977.

[24] BONAVETTI, V., DONZA, H, RAHHAL, V., IRASSAR, E., "Influence of Initial Curing on the Properties of Concrete Containig Limestone Blended Cement", Cement and Concrete Research, v. 30, pp. 703-708, 2000.

[25] SCIAN, A.N., PORTO LÓPEZ, J.M., PEREIRA, E., "Mechanochemical Activation of High Alumina Cements, Hydration Behaviour I”, Cement and Concrete Research, v. 21, pp. 51- 60, 1991.

[26] ALUMNO ROSSETTI, V., MEDICI, F., "Inertization of Toxic Metals in Cement Matrices: Effects on Hydration, Setting and Hardening”, Cement and Concrete Research, v. 5, pp. 1147-1152, 1995.

[27] MINDESS, S., YOUNG, J., Concrete, Englewood Cliffs, Prentice- Hall, 1981. 